\title{
BIOINFORMATION
}

Discovery at the interf face of physical and biological sciences

\section{Modelling and docking analysis of a tumor target protein BAG3}

\author{
Idris Mukhtar1, Suleiman Oluwaseun Alakanse ${ }^{2}$, Faoziyat Adenike Sulaiman², George Oche \\ Ambrose $^{2}$, Ohanaka Judith Nkechinyere ${ }^{3}$, Oluwaseyi Israel Malachi ${ }^{4}$ \& Adigun Adedayo ${ }^{2}$ \\ 1School of Life Sciences University of Science and Technology of China Hefei, Anhui Province, People's Republic of China; \\ ${ }^{2}$ Department of Biochemistry, University of Ilorin, Ilorin, Nigeria; ${ }^{3}$ Department of Biochemistry, Faculty of Natural and Applied \\ Sciences, Nile University of Nigeria, Nigeria; ${ }^{4}$ Food Production Department, Goodfoods Inc, Montreal, Quebec, Canada; Suleiman \\ Oluwaseun Alakanse - E-mail: oluwaseunalakanse@gmail.com; Oluwaseyi Israel Malachi - E-mail: Malachiseyi@gmail.com
}

The authors choose not to provide E-mail ID for all authors and they did not respond to PROOF corrections

Received February 8, 2020; Revised April 1, 2020; Accepted April 4, 2020; Published April 30, 2020

DOI: $10.6026 / 97320630016351$

The authors are responsible for the content of this article. The Editorial and the publisher has taken reasonable steps to check the content of the article with reference to publishing ethics with adequate peer reviews deposited at PUBLONS.

\section{Declaration on Publication Ethics:}

The authors state that they adhere with COPE guidelines on publishing ethics as described elsewhere at https:// publicationethics.org/. The authors also undertake that they are not associated with any other third party (governmental or non-governmental agencies) linking with any form of unethical issues connecting to this publication. The authors also declare that they are not withholding any information that is misleading to the publisher in regard to this article.

\section{Declaration on official E-mail:}

The corresponding author declares that official e-mail from their institution is not available for all authors

\section{Note:}

This article is from Nigeria, which is far from developing Nations as per UN classification. Hence, the author's state that they are unable to contribute to open access publication. The authors made several requests describing their inability to contribute towards open access (GOLD) publication. Biomedical Informatics $(P)$ Ltd., India, sponsors the open access charge for this article.

\begin{abstract}
:
BCL2 associated anthanogene (BAG)s are class of proteins linked to tumorigenesis and apoptosis. Therefore, it is of interest to design and develop potent inhibitors for BAG. Hence, we report the optimal structure-based docked features of sesamolin with BAG3 with the acceptable ADMET properties for consideration in the context of Glioblastoma Multiforme treatment and therapy.
\end{abstract}

Keywords: Glioblastoma, BAG3, tumorigenesis, sesamolin, apoptosis.

Background:

Glioblastoma is the most aggressive and occurring brain tumor in humans [1]. The BCL2 associated anthanogene BAG3 proteins is a survival protein that has been shown to be stimulated during cell response to stressful conditions, such as high temperatures and heavy metals [2] and have been found to be involved in the HSF/HSP70/BAG3 pathway which confer resistance in glioma cells to apoptosis [3]. BCL2 associated anthanogene (BAG) s are a 

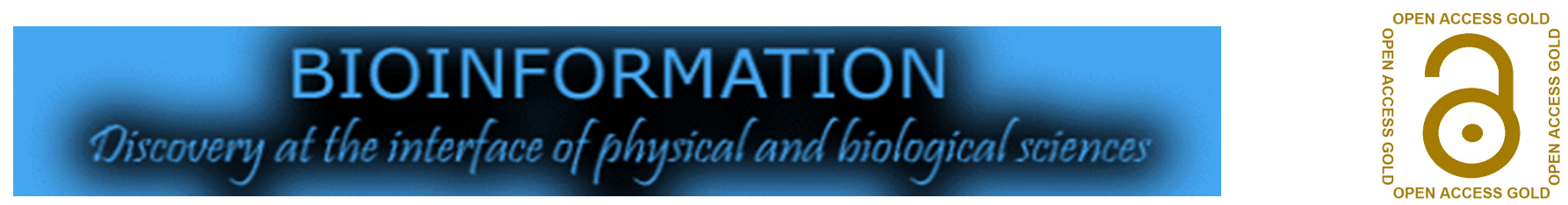

cross-functional class of proteins biochemically implicated to have varied physiological roles within the ambit of tumorigenesis and apoptosis with highly conserved genes [4]. The BCL2 associated anthanogene domains (BAG) across species are commonly conserved regions close to the $\mathrm{C}$ terminal in BAG family and this has been implicated to orchestrate interaction with the ATPase domain, which allows phosphorylation [5], located on the HSP70/HSC70 molecular chaperones [6-7]. BAG3 may regulate the chaperone activity of HSP70 [8-9] and other signalling pathways involved in cancer development through its domains [9-12]. The anti-apoptotic BAG3 protein has been found to be capable of maintaining metastatic cell survival $[13,14]$ and it's over expression can promote cell proliferation with up regulation of auto-phagy genes [15]. The Nuclear factor-kappa $B$ $\left(\mathrm{NF}_{-} \mathrm{B}\right)$-inducing kinase (NIK), in the $\mathrm{NF}_{-} \mathrm{B}$ pathway has been identified to mediate the up regulation of BAG3 which gives an explanation to rhabdomyosarcoma cells resistance to treatment [16] and this up regulation of BAG3 is not a general feature of apoptotic cells [17]. The BAG3 is up regulated in colon cancer cells [18]. The expression of BAG3 can be negatively regulated [19-20], blockage of the PI3K/AKT pathway can reduce levels of expression of BAG3 [21]. However, BAG3 knockdown was found not to interfere with the stabilization of anti-apoptosis-related proteins in retinoblastoma cells [22] and also decreases insulin cell content with increase secretion involved in diabetes pathogenesis [23]. For over 40 centuries, Sesame orientale, a potential oilseed plant, has been grown globally [24]. The $S$. orientale contains phytochemicals whose therapeutic roles have been validated both in the in vitro and in vivo studies, which further established their anti-hypertensive, anti-estrogenic, hepatoprotective, and anti-cancer properties [25-29]. Anti-cancer characteristics of Sesamin were attributed to its pro-apoptotic, anti-metastatic, anti-angiogenic, anti-inflammatory, and anti-auto phagocytic activities and thus portrayed to play critical roles in a number of signal transduction pathways that orchestrate angiogenesis, proliferation, apoptosis, and oxidative stress [15]. Pharmacological intervention by interfering with the BAG3 function to reactivate apoptotic cell death in glioma can be useful for future therapy on glioblastoma [3]. Therefore, it is of interest to design and develop potent inhibitors for BAG with improved binding features.

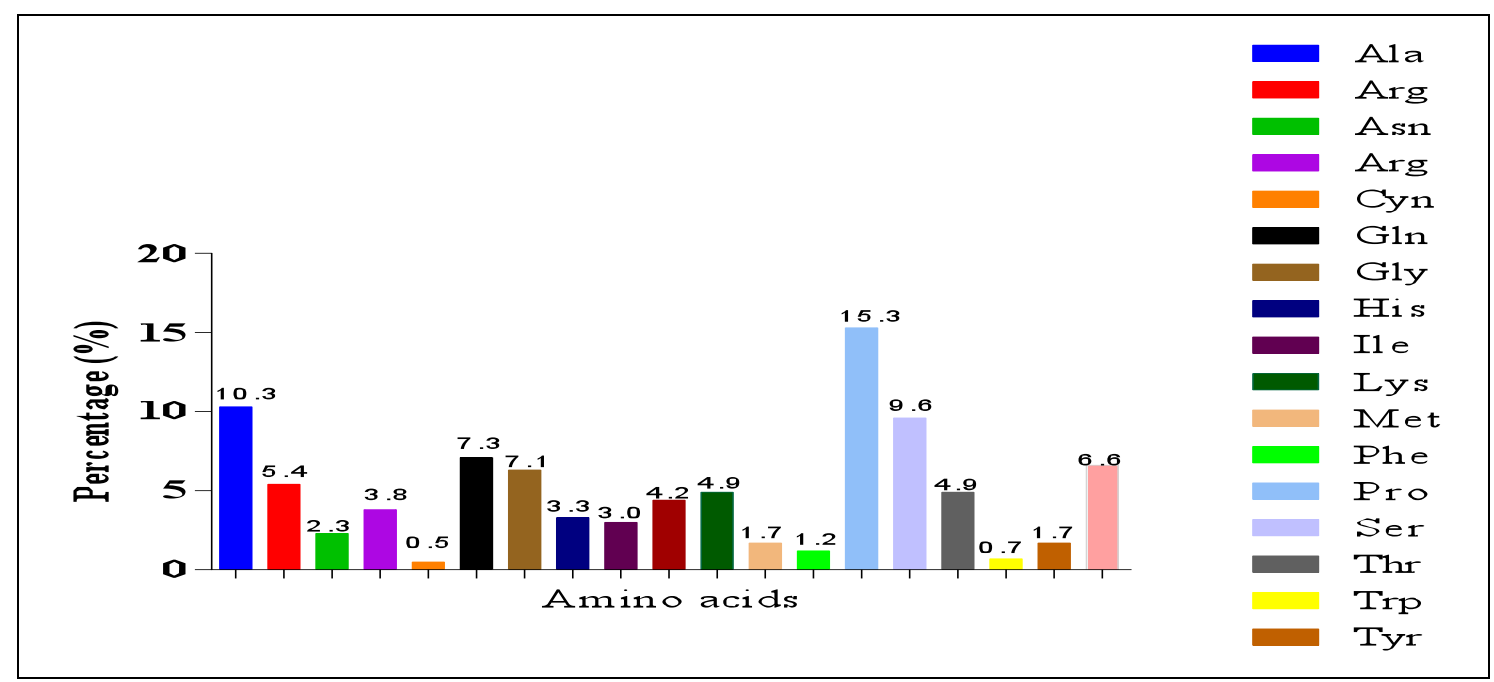

Figure 1: Composition of the 575 amino acids residues with (Pro) having the highest concentration followed by (Cyn) having the lowest concentration

Table 1: Domains in BAG3 and their amino acid sequence ranges

\begin{tabular}{llllll}
\multicolumn{6}{c}{ Table 1: Domains in BAG3 and their amino acid sequence ranges } \\
\hline \multirow{4}{*}{ Domain } & Source & ID & Start & End & Length of amino acid sequence \\
& & & & & \\
\multirow{4}{*}{ WW } & PFam & PF00397.26 & 22 & 52 & 31 \\
& SAMRT & SM00456 & 21 & 54 & 33 \\
& PROSITE & PS01159 & 26 & 52 & 27 \\
\multirow{4}{*}{ BAG } & PFam & PF02179.16 & 426 & 496 & 71 \\
& SAMRT & SM0264 & 421 & 498 & 78 \\
& PROSITE & PS51035 & 421 & 498 & 78 \\
\hline
\end{tabular}

\section{Materials and Methods:}

Ligand selection and preparation:

The twenty-nine phyto-chemicals of Sesame orientale used in this study were retrieved from FoodB (foodb.ca) [30] and PubChem compound database [31] in MOL SDF format which was converted to PDBQT file using PyRx tool to generate atomic coordinates, and energy minimization was carried out using the optimization algorithm at force field set at Universal Force Field (UFF) has required on PyRx. The standard used, Obatoclax Mesylate, a synthetic inhibitor of the bcl-2 family of proteins with potential pro-apoptotic and antineoplastic activities was also retrieved from the PubChem database and converted also to PDBQT file using PyRx tool. 


\section{BIOINFORMATION}

Table 2: Top two templates with their corresponding sequence identity

\begin{tabular}{lllllll} 
Template & Name of Template & GMQE & Identity & Method & Oligo State & Found by \\
\hline IUK5.1. A & BAG3 & 0.11 & $93.88 \%$ & NMR & Monomer & BLAST \\
IUK5.1. A & BAG3 & 0.10 & $98.77 \%$ & NMR & Monomer & BLAST \\
\hline
\end{tabular}

Table 4: Binding affinity of sesamolin, sesamin, and justisolin using AutoDock Vina scoring function algorithm.

\begin{tabular}{llll} 
Compounds & Fred Docking Score & SwissDock Score & AutoDock Vina \\
\hline Sesamolin & -4.31 & -7.39 & -6.8 \\
Sesamin & -4.0 & -6.85 & -6.8 \\
Justisolin & -3.92 & -7.24 & -6.8
\end{tabular}

Homology modeling of B-cell lymphoma 2 (Bcl-2)-associated anthanogene (BAG3) target protein:

The crystal structure of Human BAG3 was unavailable in the PDB databank; hence homology modeling was used to generate the 3D structure needed for this study. Human BAG3 protein FASTA query sequences (NP_004272.2) were retrieved from NCBI and PDB databases. The human BAG3 was subjected to the Basic Local Alignment Search Tool algorithm (BLASTP). The multiple sequence alignments (MSA) were carried out using CLUSTAL W [32] and a phylogenetic tree was constructed while the identical and similar amino acids are shaded or colored. The relevance of this technique is to analyze the sequence similarities of BAG3 across different organisms. In order to find the conserved region, for this purpose, we used the Cunsurf server (http://consurf.tau.ac.il/). Two templates with the same ID 1UK5.1.A but different sequence identity of $93.88 \%$ and $98.77 \%$ were used for homology modeling using a SWISS-MODEL web server (swisssmodel.expasy.org). These tools were used to generate a 3D structure of the homology-modeled protein.

Optimization and Refinement of B-cell lymphoma 2 (Bcl-2)associated anthanogene (BAG3) modeled protein:

The generated homology model was uploaded on 3Drefine webserver, this makes use of iterative optimization of hydrogen bonding network in addition to atomic-level energy minimization on the optimized protein model using a composite physics and knowledge-based force fields for efficient protein structure refinement [33].

Validation and quality estimation of Optimized and Refined Bcell lymphoma 2 (Bcl-2)-associated anthanogene (BAG3) modeled protein:
The optimized BAG3 model was validated using RAMPAGE, PROSESS [34], and PDBsum server [35]. The quality estimation of the modeled protein was carried out using ERRAT, VADAR, and SAVES servers, and ProSA-web. The resolution of the optimized modeled Human BAGE3 was calculated using ResProx server and visualized using Discovery Studio 3.0.

Submission of the model in the protein model database:

The model generated for human BAG3 was successfully submitted in the protein model database (PMDB) having PMDB ID: PM0082220 [36].

\section{Molecular docking using PyRx:}

For our analysis, we used the PyRx, AutoDock Vina exhaustiveness search docking function. After the minimization process, the grid box resolution of human BAG3 was centered at $1.7888 \times-6.9655 \times 0.9312$ along the $\mathrm{x}, \mathrm{y}$ and $\mathrm{z}$ center axes respectively at the grid dimension of $33.1350 \times 53.635 \times 33.906 \AA$ to define the binding site [37].

\section{Drug likeness and ADME-Toxicity:}

The Canonical smiles of the Sesamolin (PubChem CID: 101746) were gotten from the PubChem Database and were used to analyze the ADMET properties of the Sesamolin using the SwissADME server [38]. ADME (absorption, digestion, metabolism, and excretion) and toxicity (mutagenic, tumorigenic, irritant) properties determine the biological effect of drugs. Using the Variable Nearest Neighbor ADMET (vNN-ADMET) server https://vnnadmet.bhsai.org/vnnadmet/home.xhtml, sesamolin biological effects on health were determined. The vNN-ADMET web server is equipped with prebuilt ADMET models and these models assess properties like the cytotoxicity, mutagenicity, drug-drug interactions, and likelihood of causing liver injury [3940].

\section{Lead optimization:}

Validation of docking results:

The docking protocol was further validated using docking for Obloclax and the hits to the binding site of human B-cell lymphoma 2 (Bcl-2)-associated anthanogene (BAG3) modeled protein using Fred and SwissDock scoring function.

Table 3: Best model indicating 3D ${ }^{\text {refine }}$ Score, Molprobity, RMSD, RWplus, GDT-HA, and GDT-TS after energy minimization

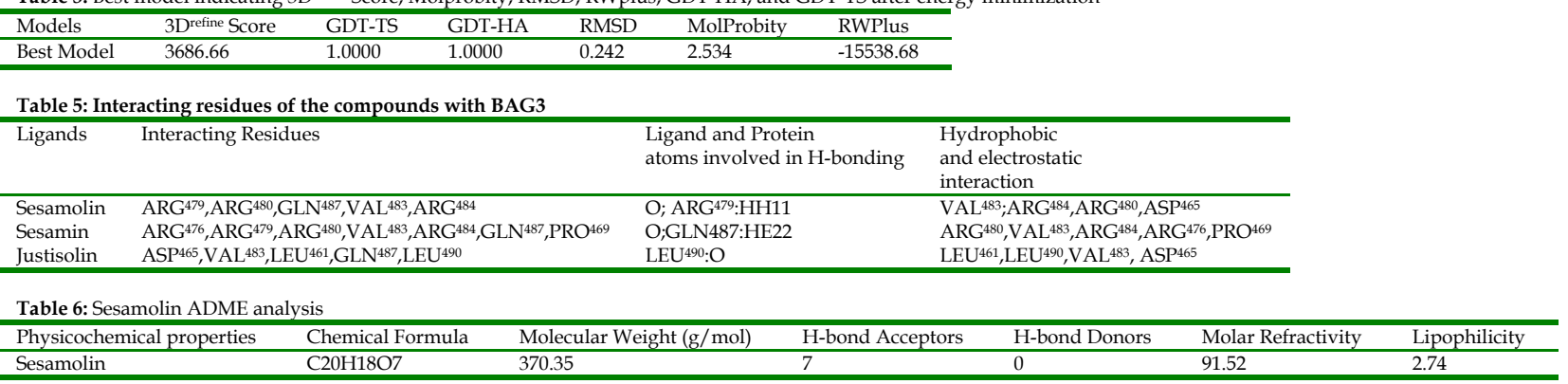




\section{BIOINFORMATION Discovery at the interf face of physical and biological sciences}

Table 7: ADME-Tox of Sesamolin using vNN-ADMET

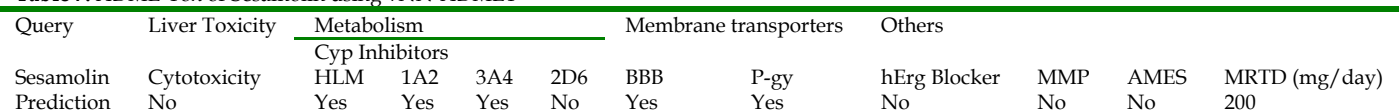

${ }^{*} \mathrm{HLM}=$ Human Liver Microsomal Stability, Cyp1A2 = Cytochrome p450 1A2, Cyp3A4 = Cytochrome p450 3A4, Cyp2D6 = Cytochrome p450 2D6, BBB = blood brain barrier, P-gp = glycoprotein, MMP = metallo matrix protein, $\mathrm{MRTD}=$ maximum recommended therapeutic dose

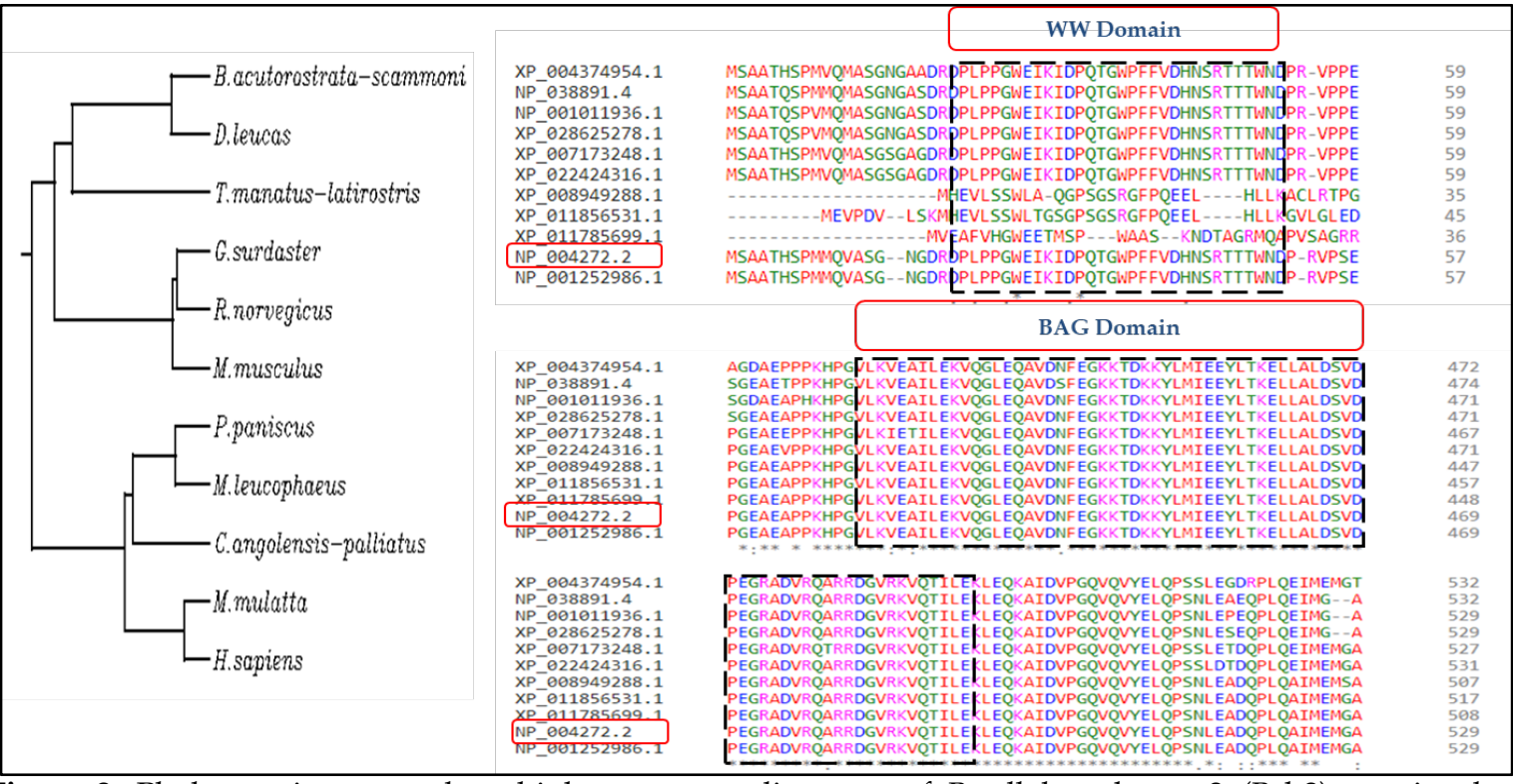

Figure 2: Phylogenetic tree and multiple sequence alignment of B-cell lymphoma 2 (Bcl-2)-associated anthanogene (BAG3) cochaperone from different species indicating the BAG and WW domain
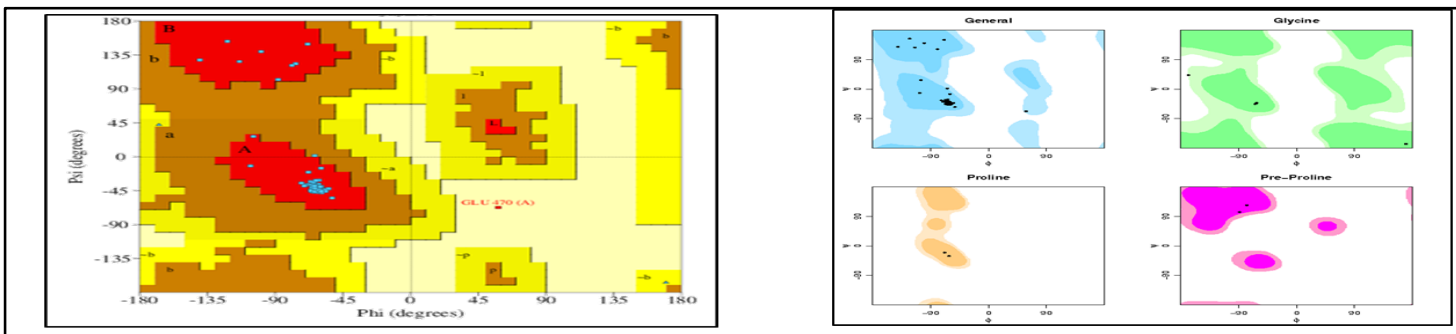

Ramachandran plots of B-cell lymphoma 2 (Bcl-2)-associated anthanogene (BAG3) co-chaperone
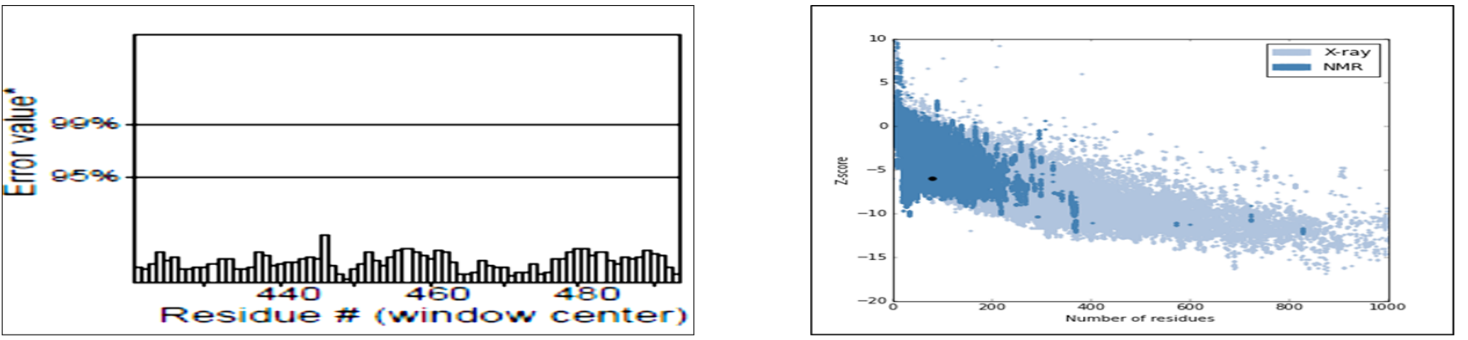

ERRAT2 plot and overall protein model quality Z-score of B-cell lymphoma 2 (Bcl-2)-associated anthanogene (BAG3) co-chaperone

Figure 3: Overall protein model quality check 


\section{BIOINFORMATION}

Discovery at the interf face of physical and biological sciences

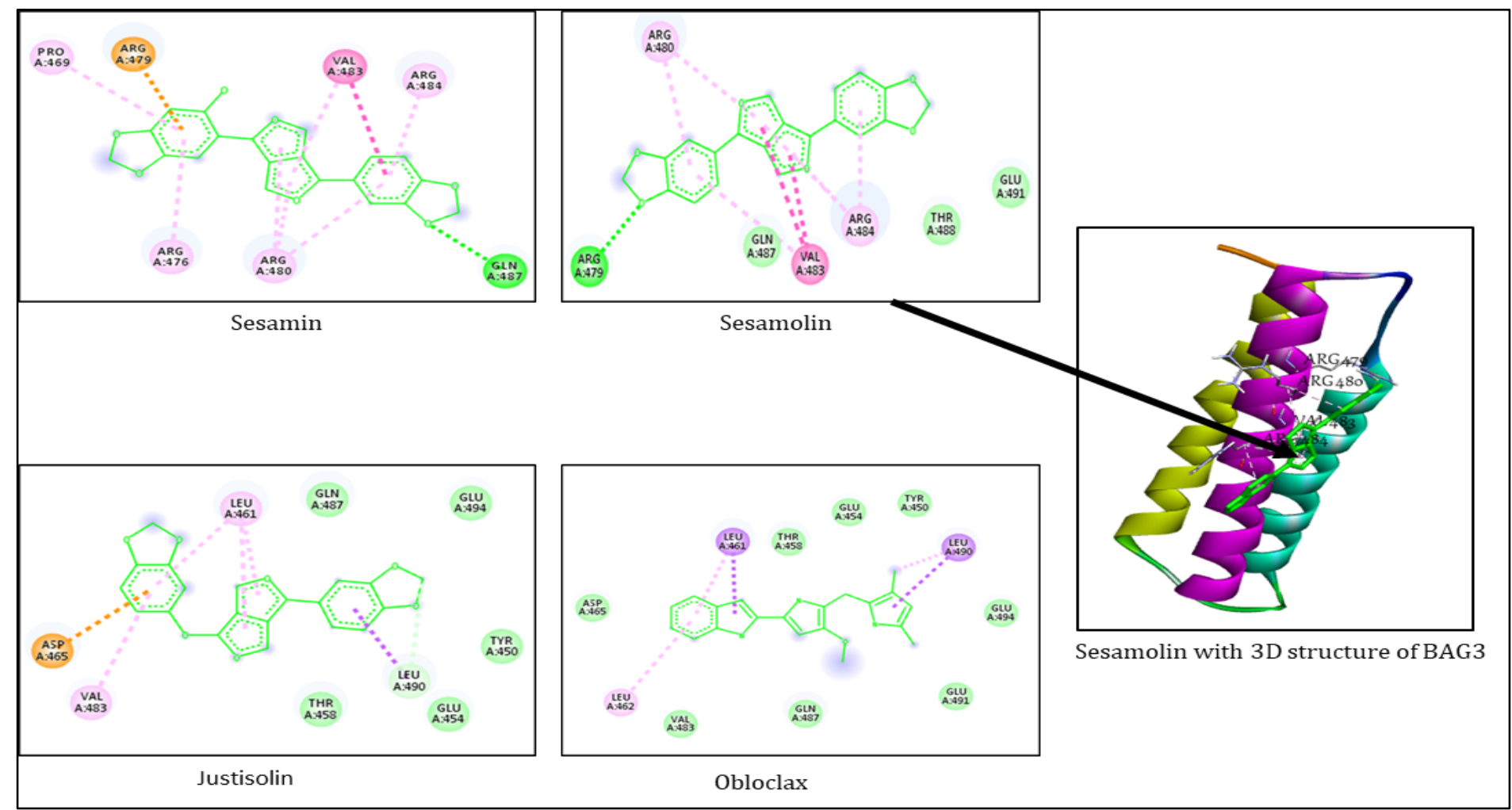

Figure 4: Sesamolin, Sesamin, Justisolin, and Obloclax (co-crystalized) ligands interaction with B-cell lymphoma 2 (Bcl-2)-associated anthanogene (BAG3) within the BAG domain

Using AutoDock Vina to study the protein-ligand binding, Sesamolin, Sesamin, and Justisolin are the lead compounds in comparison to the standard drug, which is Obloclax used in this study as indicated in figure 4. Sesamolin, Sesamin, and Justisolin all have the same docking score of $-6.8 \Delta \mathrm{G} \mathrm{kcal} / \mathrm{mol}$ compared to Obloclax which has a scoring function of $-6.4 \Delta \mathrm{G} \mathrm{kcal} / \mathrm{mol}$. But at redocking of the three leads using Fred Docking Score, and SwissDock, Sesamolin was indicated to be the consistent lead of the three as depicted in table 4 below. As indicated in figure 4, the amino acid residues VAL ${ }^{483}$; ARG484; ARG480; and ASP465 are involved in hydrophobic and electrostatic interactions. While the amino acid residues involved in other forms of interactions with sesamin at the active site of BAG3 includes, ARG480; VAL ${ }^{483}$; $\mathrm{ARG}^{484 ;} \mathrm{ARG}^{476}$ and $\mathrm{PRO}^{469}$. On the other hand, the residues involved in Justisolin hydrophobic and electrostatic interaction with the active site of BAG3 includes LEU461; LEU490; VAL483; and $\mathrm{ASP}^{465}$. Amino acid residues; $\mathrm{ARG}^{479}, \mathrm{GLN}^{487}$, and LEU 490 are the only residues involved in the formation of hydrogen bond interaction between Sesamolin, Sesamin, and Justisolin with the active site of BAG3 respectively.

The drug-likeness analysis of Sesamolin on the SwissADME shows that it doesn't violate the Lipinski's rule of five (rule of five include, Mol_W < 500, $\log \mathrm{P}<5$, Donor H-bond $\leq 5$, Acceptor Hbond $\leq 10$ and Molar Refractivity (40-130). Therefore, sesamolin is considered druglike. The ADME-Tox of sesamolin was predicted by the Variable Nearest Neighbor ADMET (vNN-ADMET) server method. The ADMET has an influence on the drug level and the kinetics of drug exposure to tissues. Sesamolin shows positive predictions for ADMET endpoints, Human Liver Microsomal (HLM) stability test, can inhibit Cyp1A2, Cyp3A4, and also glycoprotein inhibition. Sesamolin having the potential of being a therapeutic agent has a maximum recommended dose of $200 \mathrm{mg}$ per day in clinical conditions.

\section{Conclusion:}

We report the optimal structure-based docked features of Sesamolin with BAG3 with the acceptable ADMET properties for consideration in the context of Glioblastoma Multiforme therapy. 


\section{BIOINFORMATION}

\section{Discovery at the intertace of physical and biological sciences}

\section{References:}

[1] Louis et al. Acta Neuropathologica, 2007 114:97. [PMCID: PMC1929165]

[2] Du et al. Biochemical and biophysical Research Communications 2008 369:894

[3] Antonietti et al. Molecular Cancer Therapeutics, 2016 16:156.

[4] Kabbage M \& Dickman MB, 2008. [PMCID: PMC5349150]

[5] Du et al. American Journal of Translational Research 2015 7: 2805.

[6] Brive et al. 2001. Biochemical and Biophysical Research Communications, 289:1099.

[7] Varlet et al. Cell Stress and Chaperones 2017 22:553. [PMCID: PMC5465032]

[8] Kim et al. Journal of Biological Chemistry. 2013 288:28713. [PMCID: PMC3789968]

[9] Li et al. BMC cancer. 2018 18:793. [PMCID: PMC6080525]

[10] Franceschelli et al. Oxidative Medicine and Cellular Longevity 2018 1. [PMCID: PMC5908129]

[11] Colvin et al. Cancer Research 2014 74: 4731. [PMCID: PMC4174322]

[12] Galdiero et al. Oncotarget. 2018 [PMCID: PMC5814278]

[13] Mani et al. World Journal of Urology 2015 34: 197.

[14] Guerriero et al. 2017 [PMCID: PMC5655206]

[15] Zhang et al. Cellular Physiology and Biochemistry 2016 39: 491.

[16] Rapino et al. Cell Death \& Disease 2015 6: e1692. [PMCID: PMC4385908]

[17] Rapino et al. Oncogene 2013 33:1713.

[18] Wang et al. Molecular carcinogenesis 2018 57: 284.

[19] Li, X et al. British Journal of Cancer 2016 116: 66. [PMCID: PMC5220146]

[20] Das et al. Neoplasia 2018 20: 263. [PMCID: PMC5852393]

[21] Yuan et al. Oncol Lett. 2019. [PMCID: PMC6403502]

[22] Yunoki et al. Graefe's Archive for Clinical and Experimental Ophthalmology 2014 253: 399. [PMCID: PMC5453117]

[23] Iorio et al. Cell Death \& Disease 2015 6: e1684. [PMCID: PMC4385931]
[24] Shin et al. 2016. Applied Biological Chemistry, 59: 129.

[25] Makinde FM \& Akinoso R. 2013. International Food Research Journal, 20:2293.

[26] Keowkase et al. Biomedicine \& Pharmacotherapy. 2018 107: 656

[27] Nakano et al. Clinical and Experimental Pharmacology and Physiology. 2008 35: 324.

[28] Elleuch et al. Nuts and seeds in health and disease prevention 20111029

[29] Jeng KCG \& Hou RCW. Current Enzyme Inhibition. 2005 1:11.

[30] Duncan et al. Cell. 2012 149:307. [PMCID: PMC3328787]

[31] Kim et al. 2015 Nucleic acids research, 44: D1202.

[32] Larkin et al. Bioinformatics. 2007 23: 2947.

[33] Bhattacharya et al. Nucleic acids research. 2016 44: [PMCID: PMC4987902]

[34] Berjanskii et al. Nucleic acids research. 2010 38: [PMCID PMC1538894]

[35] Laskowski et al. Protein Science 2018 27: 129. [PMCID: PMC5734310]

[36] Castrignano et al. Nucleic acids research 2006 34:D306 [PMCID: PMC1347467]

[37] Trott O \& Olson A. J Journal of computational chemistry 2010 31: 455. [PMCID: PMC3041641]

[38] Daina et al. Scientific reports 2017 7: 42717. [PMCID: PMC5335600]

[39] Schyman et al. Frontiers in pharmacology, 2017 8: 889. [PMCID: PMC5722789]

[40] Villano et al. Neuro-oncology. 2009 12: 257.

[41] Behl C. Trends in pharmacological sciences 2016 37: 672.

[42] Kathage et al. Molecular Cell Research 2017 1864: 62.

[43] Macias et al. Nature 1996 382: 646.

[44] Yu et al. PloS one. 2014 9: e99368. [PMCID: PMC4049835]

[45] Sievers F \& Higgins DG Humana Press, Totowa, NJ. 2014105. [PMCID: PMC5734385]

License statement: This is an Open Access article which permits unrestricted use, distribution, and reproduction in any medium, provided the original work is properly credited. This is distributed under the terms of the Creative Commons Attribution License 


\section{BIOINFORMATION}

Discovery at the interface of physical and biological sciences

Articles published in BIOINFORMATION are open for relevant post publication comments and criticisms, which will be published immediately linking to the original article for FREE of cost without open access charges. Comments should be concise, coherent and critical in less than 1000 words. 


\section{BIOINFORMATION}

Discovery at the interface of physical and biological sciences

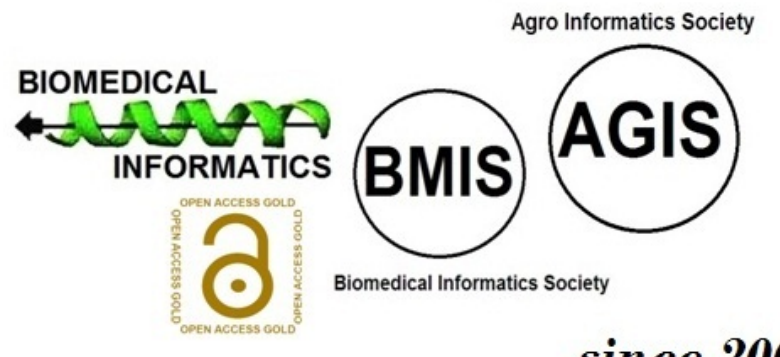

since 2005

\section{BIOINFORMATION}

Discovery at the interface of physical and biological sciences

\section{indexed in}

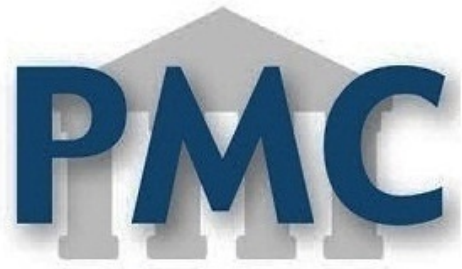

PublMed

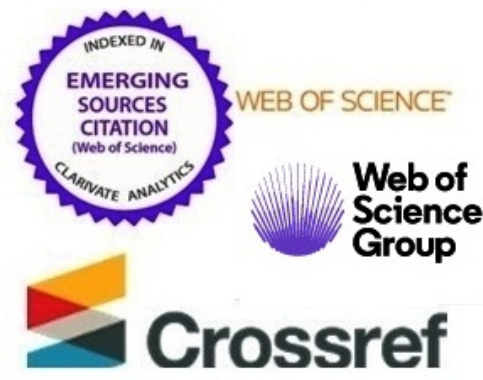

EBSCO

Web of

Science

\section{ResearchGate}
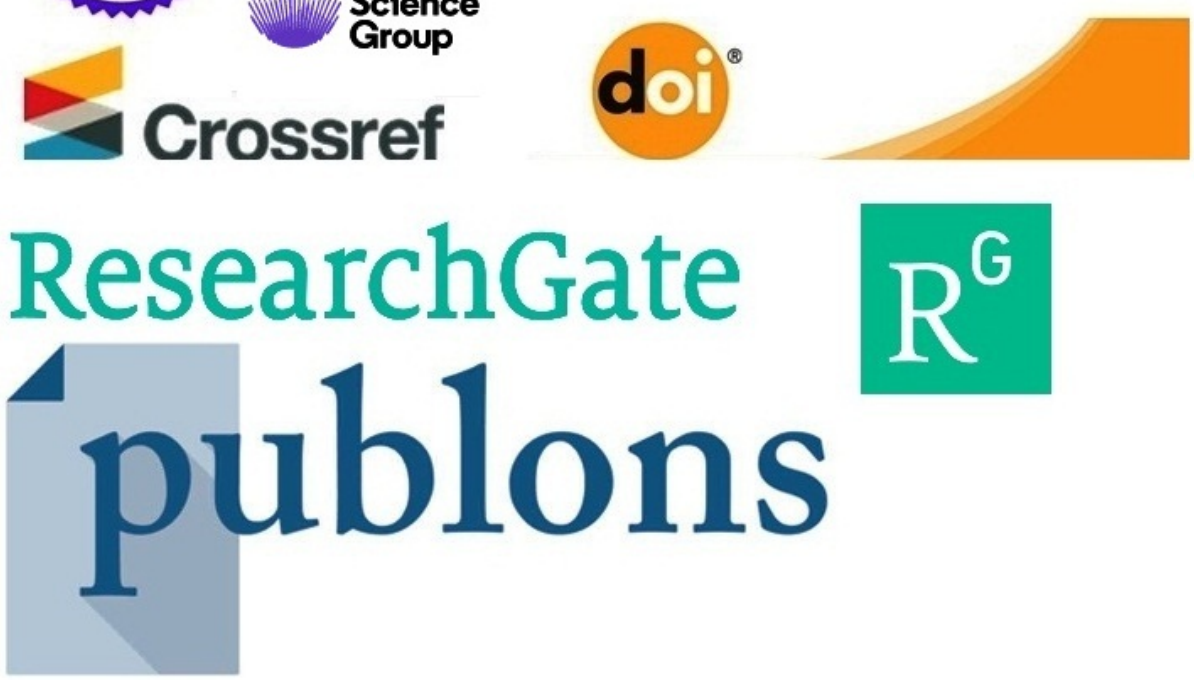